\title{
Omission of Adjuvant Chemotherapy in Rectal Cancer Patients with Pathologic Complete Response: a National Analysis
}

\author{
Mackenzie C. Morris ${ }^{1}$ (D) $\cdot$ Leah K. Winer $^{1} \cdot$ Tiffany C. Lee $^{1} \cdot$ Shimul A. Shah $^{1} \cdot$ Janice F. Rafferty $^{1} \cdot$ Ian M. Paquette $^{1}$
}

Received: 19 May 2020 / Accepted: 16 July 2020 / Published online: 29 July 2020

(C) 2020 The Society for Surgery of the Alimentary Tract

\begin{abstract}
Background An increasing number of patients achieve a pathologic complete response (pCR) after neoadjuvant chemoradiation for locally advanced rectal cancer. Consensus guidelines continue to recommend oncologic resection followed by adjuvant chemotherapy in these patients. We hypothesize that there is significant variability in compliance with this recommendation.

Methods The National Cancer Database was queried from 2006 to 2015 for patients with locally advanced rectal cancer who underwent neoadjuvant chemoradiation followed by oncologic resection with a pCR (ypT0N0). Hierarchical logistic regression models were used to generate risk and reliability-adjusted rates of adjuvant chemotherapy utilization in patients with pCR at each hospital.

Results In total, 2421 pCR patients were identified. Five-year overall survival was improved in pCR patients who received adjuvant chemotherapy compared with those who did not $(92$ vs. $85 \%, p<0.01)$. Multivariate analysis indicated that improvement in overall survival remained associated with adjuvant chemotherapy (HR $0.60,95 \%$ CI $0.44-0.82, p<0.01$ ). The mean adjuvant chemotherapy utilization rate among hospitals was $32 \%$. There was an upward trend in use over the past decade, but two-thirds still do not receive the recommended therapy. High chemotherapy utilizer hospitals were more likely to be academic centers ( 54.9 vs. $45.9 \%, p<0.01)$ when compared with low chemotherapy utilizers.

Conclusion Adjuvant chemotherapy is associated with improved survival in rectal cancer patients with pCR following neoadjuvant chemoradiation and oncologic resection. However, utilization among centers in the USA was only $32 \%$ with significant variability across centers. National efforts are needed to standardize treatment patterns according to national guidelines.
\end{abstract}

Keywords Rectal adenocarcinoma $\cdot$ Total mesorectal excision $\cdot$ Adjuvant chemotherapy $\cdot$ Pathologic complete response

\section{Introduction}

The management of patients with adenocarcinoma of the rectum has changed over the past two decades, and though new approaches such as total neoadjuvant therapy (TNT) are being studied, the current standard for locally advanced rectal

\section{Presentation}

This manuscript was accepted as a Quick Shot of Distinction presentation at the 2020 American Society of Colon and Rectal Surgeons Annual meeting previously scheduled to be held in Boston, MA, from June 6 to 10,2020 . This meeting was held virtually due to the COVID-19 pandemic.

Ian M. Paquette

ian.paquette@uc.edu

1 Department of Surgery, College of Medicine, University of Cincinnati, 2123 Auburn Ave \#524, Cincinnati, OH 45219, USA cancers is neoadjuvant long-course radiation plus chemotherapy, followed by total mesorectal excision (TME). National guidelines from the National Comprehensive Cancer Network (NCCN) and the American Society of Colon and Rectum Surgeons recommend that TME surgery should be followed by adjuvant systemic chemotherapy. ${ }^{1,2}$ One population of interest is patients who achieve a pathologic complete response (pCR) after neoadjuvant therapy. Pathologic complete response rates following neoadjuvant chemoradiation vary in the literature, from around $15-20 \%$ up to $38 \%$ in a recent randomized controlled trial. ${ }^{3,4}$ Two phase III trials were unable to identify a significant difference in disease-free (DFS) or overall survival (OS) with the addition of adjuvant chemotherapy in locally advanced rectal cancer. However, both of these studies were closed early due to poor accrual and were not focused on patients with $\mathrm{pCR} .{ }^{5,6}$ Though there is some controversy as to whether these patients should be followed with a "watch and wait" strategy, most would currently 
recommend an oncologic resection in the setting of an apparent clinical complete response (cCR) ${ }^{7,8}$ The decision as to whether to administer adjuvant chemotherapy to these patients remains controversial. Two retrospective studies utilizing the National Cancer Database (NCDB), from 2006 to 2011, demonstrated a survival benefit with adjuvant chemotherapy in the setting of pCR. ${ }^{9,10}$

Given these discrepant findings, we used a contemporary cohort of the NCDB to evaluate outcomes in patients with locally advanced rectal cancer who underwent neoadjuvant chemoradiation followed by oncologic resection with a pCR. We hypothesized that there would be poor national compliance with the use of adjuvant chemotherapy in this subset of patients. We also hypothesized that patients who underwent adjuvant chemotherapy would demonstrate a survival advantage.

\section{Materials and Methods}

\section{Data Source}

The NCDB is an oncology database that consists of patient data from Commission on Cancer-accredited hospitals. The data represents over 34 million historical records and captures more than $70 \%$ of newly diagnosed cancer cases. This data includes patient demographics, facility characteristics, cancerspecific variables, surgical variables, and survival outcomes. ${ }^{1}$ This study was approved by the University of Cincinnati Institutional Review Board (IRB \#2019-0179).

\section{Patient Cohort}

The NCDB Participant User File (PUF) was queried for all patients with a diagnosis of clinical T3-4 rectal adenocarcinoma and no evidence of metastatic disease from 2006 to 2015 $(n=70,891)$ (Fig. 1). We subsequently excluded patients who did not undergo neoadjuvant chemoradiation and curative resection, who died within the first 90 days after surgery, or who did not receive 40 to 70.2 Gy of radiation. We then identified only patients who had a pCR on final pathology $(n=2421)$. These patients were then divided into groups based on whether they received adjuvant chemotherapy.

\section{Study Variables}

The following data was collected for all patients who met inclusion criteria: age, race, insurance status, education level (percentage of people who did not graduate from high school in the patient's zip code), income (median income in the patient's zip code), distance traveled for cancer care, facility type (academic $>500$ new cancer diagnoses annually, at least four postgraduate training programs; comprehensive community $>500$ new cancer diagnoses annually, postgraduate training optional; community 100-500 new cancer diagnoses annually, postgraduate training optional, integrated network cancer program; no minimum caseload requirement, postgraduate training optional), and Charlson-Deyo score. ${ }^{11,12}$ TNT was defined as any patient that received radiotherapy and multiagent chemotherapy prior to oncologic resection, RT was started a minimum of 2 months after chemotherapy, and no adjuvant chemotherapy was given. ${ }^{13}$ Cancer-specific and treatment-specific variables were also collected. Hospital volume was determined by calculating the total number of surgical cases in the rectum NCDB PUF for each facility. Facilities were then divided into the top third (high volume), middle third (medium volume), and lower third (low volume).

\section{Statistical Analysis}

All variables are represented as median (interquartile range) or percentage. Variables were compared by Pearson's chi-square test or Wilcoxon rank-sum test. Kaplan-Meier and Cox proportional hazards regression analyses were used to determine the association with OS. We utilized empirical Bayes techniques to perform reliability adjustment in order to account for differences across hospitals in use of adjuvant therapy due to chance alone. This technique is used to filter out statistical noise, particularly from hospitals which have lower numbers of cases and uses the reliability of the measurement to adjust the observed rate closer to the mean. ${ }^{14-17}$ Stepwise logistic regression was used to identify patient, cancer, and hospitalspecific factors associated with the administration of adjuvant chemotherapy. Hierarchical logistic regression models were created using the "meqrlogit" command to model variation. Post-estimation commands were utilized to create empirical Bayes estimates for each hospital's random effect. This random effect was then added to the average patient risk of receiving adjuvant chemotherapy. The inverse logit analysis was performed to calculate risk and reliability-adjusted adjuvant chemotherapy use for each hospital. Individual hospitals were then ranked based on adjuvant chemotherapy use. The top and bottom $10 \%$ of hospitals were defined as high utilization hospitals (HUH) and low utilization hospitals (LUH), respectively. A $p$ value of less than 0.05 was considered statistically significant. All statistical analyses were performed with SAS 9.4 (SAS Institute, Cary, NC) and Stata 13.1 (College Station, TX).

\section{Results}

Demographic and clinicopathologic differences between pCR patients who did and did not receive adjuvant chemotherapy were seen (Table 1). Patients who had adjuvant chemotherapy were more likely to be young, Caucasian women. This group also consisted of a higher 
Fig. 1 Consort diagram demonstrating inclusion and exclusion criteria to get the study cohort

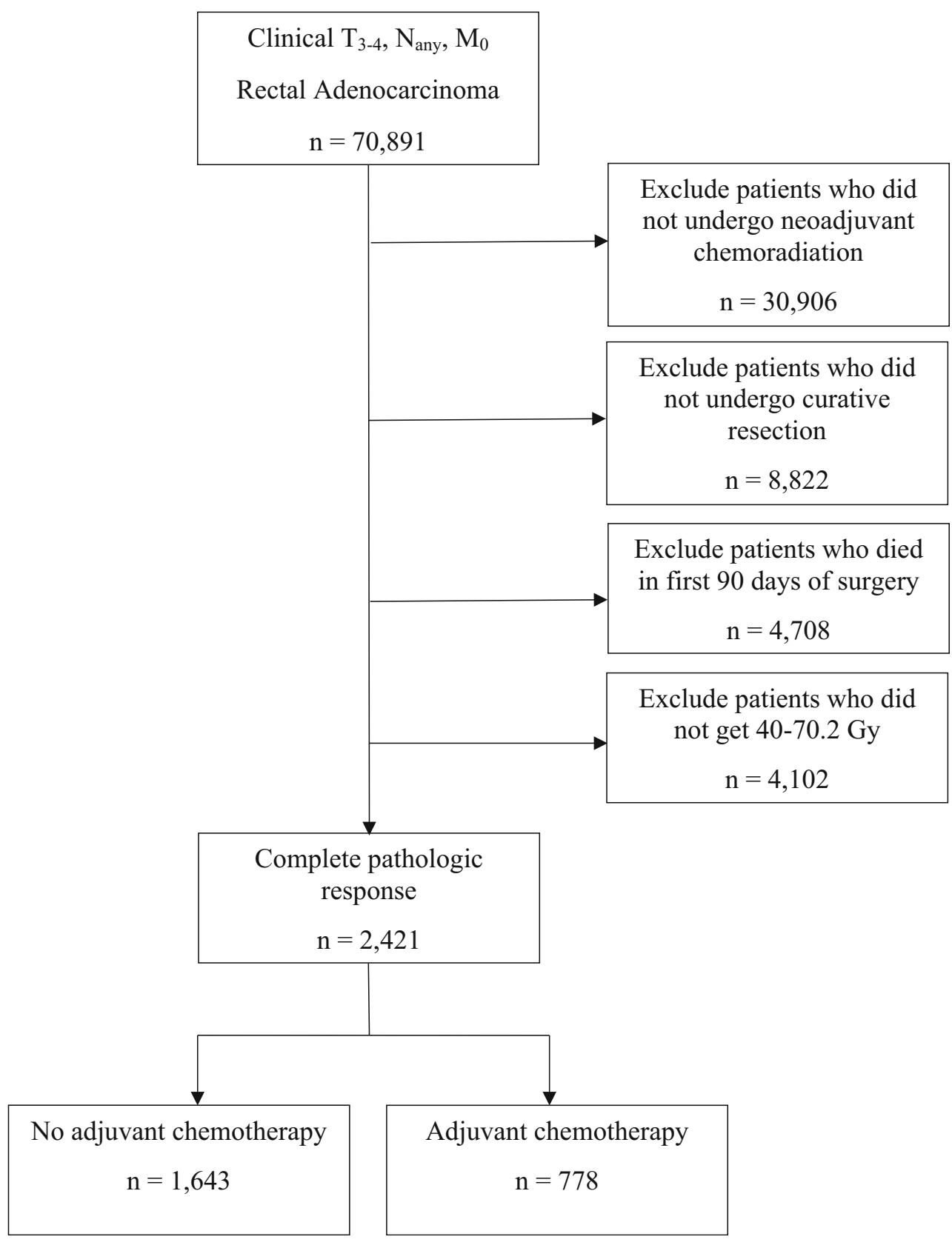

proportion of privately insured patients from better educated areas that were more likely to be treated at high volume, academic centers. They also had a greater median number of lymph nodes examined and higher proportion of positive nodal disease on the initial staging before neoadjuvant therapy. There were no differences in Charlson Comorbidity score, facility type, income, distance traveled, tumor grade or size, lymphovascular invasion, unplanned readmission within 30 days, or surgical procedure between groups. The use of adjuvant chemotherapy in this population has seen an upward trend over the past decade. However, adjuvant chemotherapy is still omitted in approximately two-thirds of patients (Fig. 2).
We next analyzed adjuvant chemotherapy outcomes and predictors of survival. The median follow-up for the entire cohort was 42.3 months and was similar in patients that did or did not receive adjuvant chemotherapy (42.7 vs. 42.0 months, $p=0.63$, respectively). Kaplan-Meier survival analysis showed a significant survival benefit with the use of adjuvant chemotherapy (5-year OS 92 vs. $85 \%, p<0.01$ ) (Fig. 3). Cox proportional regression univariate analysis found that female gender and adjuvant chemotherapy were associated with a protective effect. Older age, Medicaid insurance type, higher Charlson-Deyo score, and initial clinical stage T4 were associated with worse OS. In multivariate analysis, female gender and adjuvant chemotherapy remained 
Table 1 Demographics of patients receiving adjuvant chemotherapy and those who did not

\begin{tabular}{|c|c|c|c|}
\hline & Adjuvant chemotherapy & No adjuvant chemotherapy & $p$ value \\
\hline \multicolumn{4}{|l|}{ Patient demographics } \\
\hline Age & $58(50-66)$ & $62(53-71)$ & $<0.01$ \\
\hline Sex & & & $<0.01$ \\
\hline Male & $425(56.9 \%)$ & $997(63.1 \%)$ & \\
\hline Female & $322(43.1 \%)$ & $584(36.9 \%)$ & \\
\hline Race & & & $<0.05$ \\
\hline White & $660(88.6 \%)$ & $1358(86.1 \%)$ & \\
\hline Black & $40(5.4 \%)$ & $130(8.2 \%)$ & \\
\hline Other & $45(6.0 \%)$ & $90(5.7 \%)$ & \\
\hline Insurance status & & & $<0.01$ \\
\hline Not insured & $27(3.6 \%)$ & $48(3.1 \%)$ & \\
\hline Private insurance & $456(61.5 \%)$ & $768(49.1 \%)$ & \\
\hline Medicaid & $50(6.7 \%)$ & $90(5.8 \%)$ & \\
\hline Medicare & $206(27.8 \%)$ & $630(40.3 \%)$ & \\
\hline Other government & $3(0.4 \%)$ & $28(1.8 \%)$ & \\
\hline Education & & & $<0.01$ \\
\hline$\geq 21 \%$ & $87(11.7 \%)$ & $265(16.8 \%)$ & \\
\hline $13-20.9 \%$ & $179(24.0 \%)$ & $405(25.7 \%)$ & \\
\hline $7-12.9 \%$ & $249(33.4 \%)$ & $500(31.7 \%)$ & \\
\hline$<7 \%$ & $230(30.9 \%)$ & $407(25.8 \%)$ & \\
\hline Facility type & & & 0.19 \\
\hline Community cancer program & $28(3.9 \%)$ & $70(4.6 \%)$ & \\
\hline Comprehensive community cancer program & $273(38.3 \%)$ & $583(38.5 \%)$ & \\
\hline Academic program & $316(44.4 \%)$ & $617(40.7 \%)$ & \\
\hline Integrated network cancer program & $95(13.3 \%)$ & $246(16.2 \%)$ & \\
\hline Hospital volume & & & $<0.01$ \\
\hline Low & $25(3.4 \%)$ & $63(4.0 \%)$ & \\
\hline Medium & $104(13.9 \%)$ & $303(19.2 \%)$ & \\
\hline High & $618(82.7 \%)$ & $1215(76.9 \%)$ & \\
\hline \multicolumn{4}{|l|}{ Cancer-related characteristics } \\
\hline \# of lymph nodes examined & $14(10-18)$ & $13(9-17)$ & $<0.01$ \\
\hline Clinical T stage & & & 0.36 \\
\hline 3 & $744(95.6 \%)$ & $1557(94.8 \%)$ & \\
\hline 4 & $34(4.4 \%)$ & $86(5.2 \%)$ & \\
\hline Clinical N stage & & & $<0.01$ \\
\hline 0 & $353(45.5 \%)$ & $880(54.0 \%)$ & \\
\hline 1 & $366(47.2 \%)$ & $640(39.3 \%)$ & \\
\hline 2 & $57(7.4 \%)$ & $109(6.7 \%)$ & \\
\hline Clinical $\mathrm{T}$ and $\mathrm{N}$ stage & & & $<0.01$ \\
\hline T3N0 & $344(44.3 \%)$ & $845(51.9 \%)$ & \\
\hline $\mathrm{T} 3 \mathrm{~N} 1$ & $343(44.2 \%)$ & $607(37.3 \%)$ & \\
\hline T3N2 & $55(7.1 \%)$ & $92(5.7 \%)$ & \\
\hline T4N0 & $9(1.2 \%)$ & $35(2.2 \%)$ & \\
\hline T4N1 & $23(3.0 \%)$ & $33(2.0 \%)$ & \\
\hline T4N2 & $2(0.3 \%)$ & $17(1.0 \%)$ & \\
\hline \multicolumn{4}{|l|}{ Treatment characteristics } \\
\hline Days from diagnosis to definitive surgical procedure & $133(119-147)$ & $139(123-160)$ & $<0.01$ \\
\hline Surgical procedure & & & 0.30 \\
\hline Partial proctectomy, NOS & $507(67.9 \%)$ & $1041(65.8 \%)$ & \\
\hline Pull through with sphincter preservation & $70(9.4 \%)$ & $127(8.0 \%)$ & \\
\hline Total proctectomy & $147(19.7 \%)$ & $352(22.3 \%)$ & \\
\hline
\end{tabular}


Table 1 (continued)

\begin{tabular}{|c|c|c|c|}
\hline & Adjuvant chemotherapy & No adjuvant chemotherapy & $p$ value \\
\hline Total proctocolectomy, NOS & $15(2.0 \%)$ & $28(1.8 \%)$ & \\
\hline Proctectomy or proctocolectomy with resection of other organs & $5(0.7 \%)$ & $20(1.3 \%)$ & \\
\hline Proctectomy, NOS & $3(0.4 \%)$ & $13(0.8 \%)$ & \\
\hline Hospital length of stay & $5(4-7)$ & $6(4-8)$ & $<0.01$ \\
\hline Days from diagnosis to radiation & $34(26-45)$ & $36(27-49)$ & $<0.01$ \\
\hline Radiation dose (cGy) & $4500(4500-4500)$ & $4500(4500-4680)$ & 0.03 \\
\hline Days of radiation treatment & $39(38-42)$ & $40(38-43)$ & $<0.01$ \\
\hline Days from diagnosis to chemotherapy & $34(24-45)$ & $35(26-48)$ & 0.06 \\
\hline
\end{tabular}

Income, distance traveled, Charlson-Deyo score, tumor grade and size, lymphovascular invasion, readmission within 30 days, and type of radiation were analyzed but not different between groups

associated with improved OS. Older age, higher CharlsonDeyo score, and clinical stage T4 were predictive of worse OS (Table 2).

Finally, we sought to delineate adjuvant chemotherapy utilization patterns among U.S. institutions. The median overall risk and reliability-adjusted adjuvant chemotherapy rate among hospitals was 32\% (Fig. 4). Even at the highest utilization hospitals, the risk-adjusted rate of adjuvant chemotherapy only approached $60 \%$. Patients treated at HUH were more likely to have a high school degree. HUH were more likely to be located in the Midwest and West compared with LUH. HUH consisted of a greater proportion of academic centers and lower proportion of integrated network cancer programs. Compared with patients treated at LUH, those treated at HUH were more likely to be uninsured and better educated ( $p<0.05$ each) (Fig. 5a-d). There were no differences in hospital surgical volume or patient income between HUH and LUH.

\section{Discussion}

In this study, we utilized the NCDB to evaluate the use of adjuvant chemotherapy in patients with locally advanced rectal cancer who underwent neoadjuvant chemoradiation and oncologic resection with a $\mathrm{pCR}$. We found significantly improved overall survival in $\mathrm{pCR}$ patients who received adjuvant chemotherapy. However, $68 \%$ of all patients with $\mathrm{pCR}$ did not actually receive adjuvant chemotherapy, despite consensus guidelines. Hospitals that were high utilizers of adjuvant chemotherapy were more likely to be academic centers.

Similar to our study, other retrospective, propensitymatched studies utilizing the NCDB have demonstrated improved overall survival in patients who underwent adjuvant chemotherapy following a pCR. ${ }^{18,19}$ Polanco et al. showed a 5 -year OS near $95 \%$ in the patients who received adjuvant chemotherapy compared with $88 \%$ in the patients who did not $(p<0.01)$. In subgroup analysis, patients with T3 or T4
Fig. 2 Adjuvant chemotherapy use by year during the study period

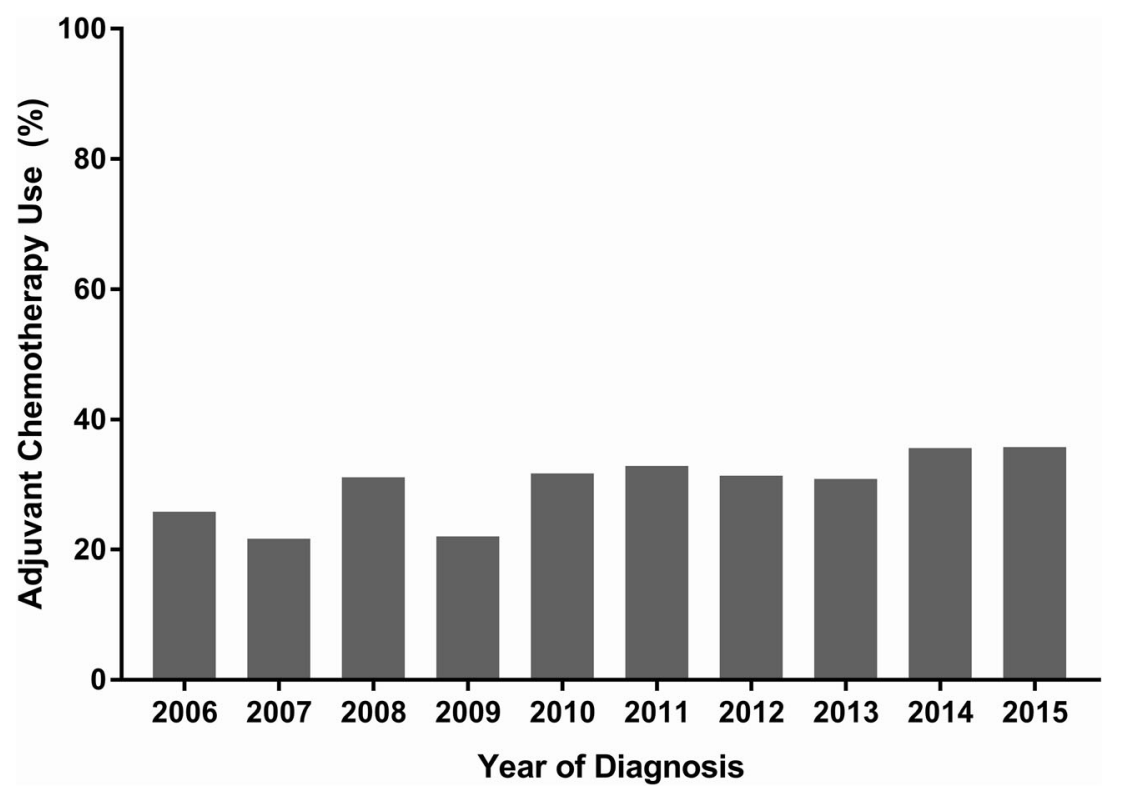


Fig. 3 Kaplan-Meier survival analysis demonstrated a significant survival benefit with the use of adjuvant chemotherapy (5-year survival 92 vs. $85 \%$, $p<0.01)$

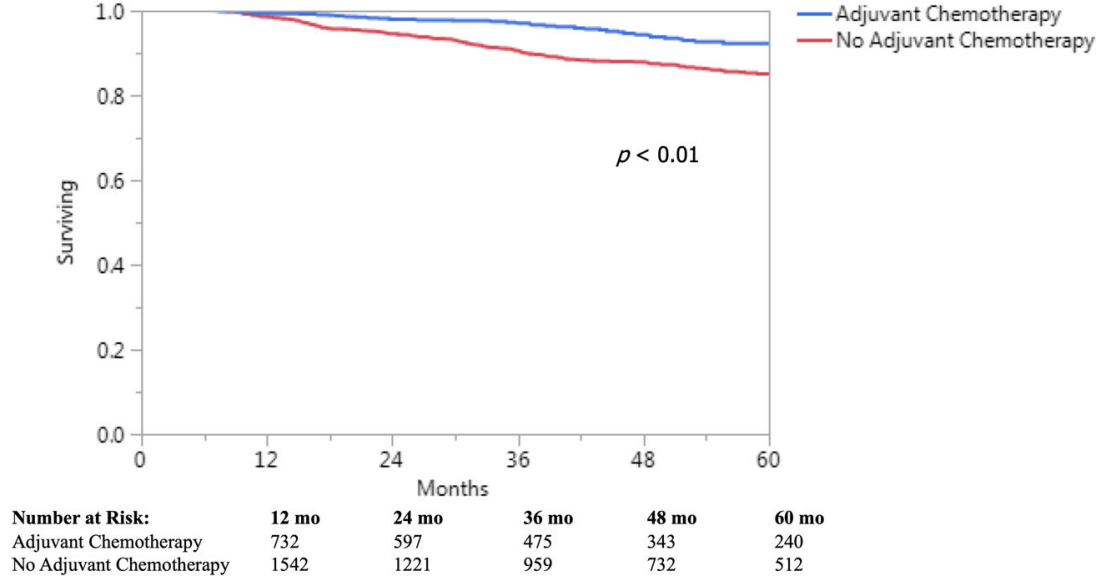

tumors and those with positive nodal disease prior to treatment received the greatest benefit from adjuvant chemotherapy. ${ }^{19}$ In contrast to these studies, a randomized controlled trial by the Dutch Colorectal Cancer Group failed to show a benefit of adjuvant chemotherapy in patients with locally advanced rectal cancer. The 5-year OS was $79.2 \%$ in the observation group and $80.4 \%$ in the adjuvant chemotherapy group. However, the study closed prior to its planned accrual and was likely underpowered to detect a difference between the groups. They also did not specifically examine patients with larger tumors or
pCR following neoadjuvant chemoradiation and resection. ${ }^{6}$ In a recently published retrospective study, Voss et al. identified over 800 patients who were diagnosed with stage 2 or 3 rectal cancer and underwent neoadjuvant chemoradiotherapy followed by total mesorectal excision at 15 centers in California. They were unable to demonstrate a benefit in recurrence free survival, their primary outcome, with the use of adjuvant chemotherapy. However, similar to the trials previously discussed, this study was not powered to examine patients with $\mathrm{pCR} .{ }^{20}$ In a Cochrane review that evaluated the use
Table 2 Univariate and multivariate Cox proportional hazards survival analysis

\begin{tabular}{|c|c|c|c|c|}
\hline \multirow[b]{2}{*}{ Variable } & \multicolumn{2}{|l|}{ Univariate } & \multicolumn{2}{|l|}{ Multivariate } \\
\hline & Hazard ratio $(95 \% \mathrm{CI})$ & $p$ value & Hazard ratio $(95 \% \mathrm{CI})$ & $p$ value \\
\hline Age & $1.05(1.04-1.06)$ & $<0.01$ & $1.04(1.02-1.05)$ & $<0.01$ \\
\hline Female gender & $0.63(0.49-0.82)$ & $<0.01$ & $0.66(0.51-0.86)$ & $<0.01$ \\
\hline \multicolumn{5}{|l|}{ Insurance type } \\
\hline Private insurance & Ref & --- & Ref & --- \\
\hline Medicare & $1.13(0.67-1.93)$ & 0.64 & $1.10(0.65-1.88)$ & 0.72 \\
\hline Medicaid & $2.68(2.08-3.47)$ & $<0.01$ & $1.22(0.87-1.74)$ & 0.25 \\
\hline Other government insurance & $2.57(0.94-7.00)$ & 0.07 & $2.10(0.77-5.75)$ & 0.15 \\
\hline \multicolumn{5}{|l|}{ Hospital volume } \\
\hline High volume & Ref & --- & Ref & --- \\
\hline Medium volume & $1.28(0.95-1.72)$ & 0.10 & $1.05(0.78-1.41)$ & 0.75 \\
\hline Low volume & $1.60(0.89-2.86)$ & 0.12 & $1.55(0.84-2.85)$ & 0.16 \\
\hline \multicolumn{5}{|l|}{ Charlson-Deyo Score } \\
\hline 0 & Ref & --- & Ref & --- \\
\hline 1 & $1.59(1.20-2.11)$ & $<0.01$ & $1.40(1.05-1.87)$ & 0.02 \\
\hline 2 & $2.17(1.30-3.62)$ & $<0.01$ & $1.58(0.94-2.67)$ & 0.08 \\
\hline $3+$ & $4.04(2.30-7.11)$ & $<0.01$ & $2.85(1.60-5.07)$ & $<0.01$ \\
\hline Initial clinical stage $\mathrm{T} 4$ & $3.27(1.83-5.86)$ & $<0.01$ & $2.63(1.44-4.79)$ & $<0.01$ \\
\hline Adjuvant chemotherapy & $0.47(0.35-0.64)$ & $<0.01$ & $0.60(0.44-0.82)$ & $<0.01$ \\
\hline
\end{tabular}

Race, education level, income, tumor grade, clinical nodal stage, lymphovascular invasion, type of surgical procedure, distance of travel, radiation dose, and time from diagnosis to starting chemotherapy/radiation were not included in the model due to $p$ value $>0.05$ 
Fig. 4 The median overall riskadjusted adjuvant chemotherapy rate among hospitals was $32 \%$ that is depicted by the dashed line

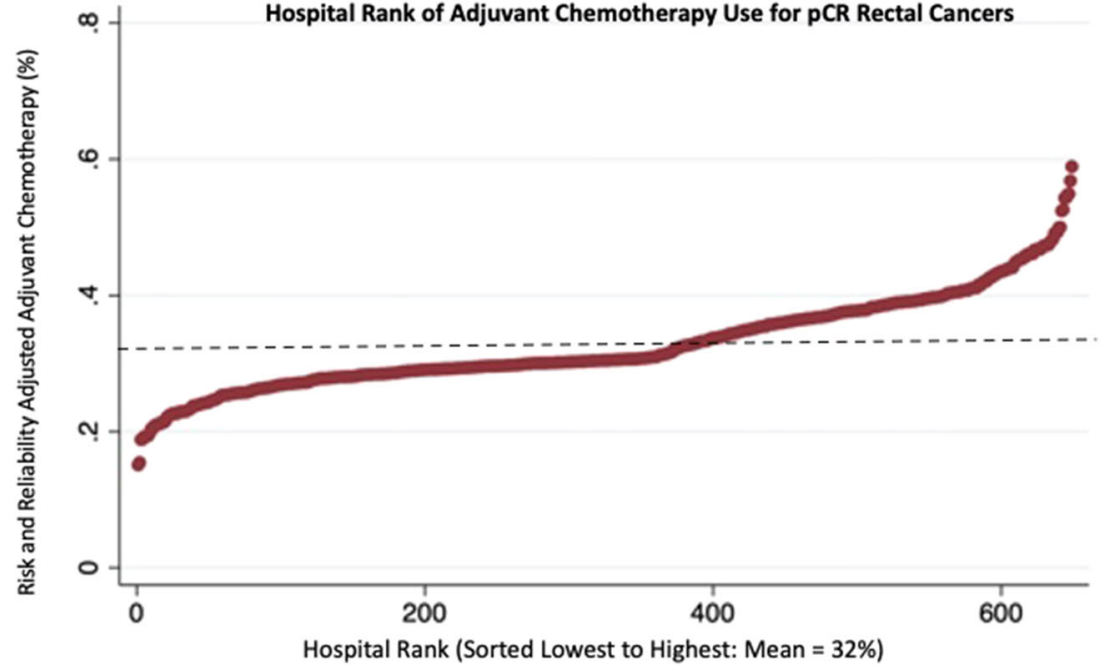

of adjuvant chemotherapy in rectal cancer in 21 eligible trials, the authors demonstrated improved survival in those receiving adjuvant chemotherapy. However, only two trials included neoadjuvant therapy, and they did not specifically look at utilization in locally advanced disease or pCR patients. ${ }^{21}$ The currently ongoing Chinese ACRNaCT trial is comparing observation with adjuvant 5-fluorouracil alone in patients with a pCR. Hopefully, this trial will add to the growing body of literature describing whether adjuvant chemotherapy offers a survival advantage beyond neoadjuvant chemoradiation and oncologic resection alone for pCR patients. ${ }^{22}$

Given that at least some evidence from population studies shows a benefit, NCCN guidelines currently recommend adjuvant chemotherapy for all patients with stage II or III rectal cancer after neoadjuvant chemoradiation and TME. ${ }^{23}$
However, our study demonstrates a slow trend of increased adjuvant chemotherapy use, but the compliance rate remains an alarming $36 \%$ in 2015 , suggesting that U.S. practice patterns deviate greatly from published recommendations. Similarly, a previous NCDB study by Xu et al. demonstrated an adjuvant chemotherapy rate of $19 \%$ in all patients with stage II or III rectal cancer, regardless of final pathology. The authors also showed that patients with pCR benefitted from adjuvant therapy, perhaps even more than patients without $\mathrm{pCR} .{ }^{10}$ Our study, distinct from prior literature, spans a more contemporary time period, captures a more diverse picture of current management, and is the first to identify a trend to forgo adjuvant chemotherapy in patients with $\mathrm{pCR}$.

In an effort to understand the immense national variability in practice patterns, we identified the top and bottom $10 \%$ of
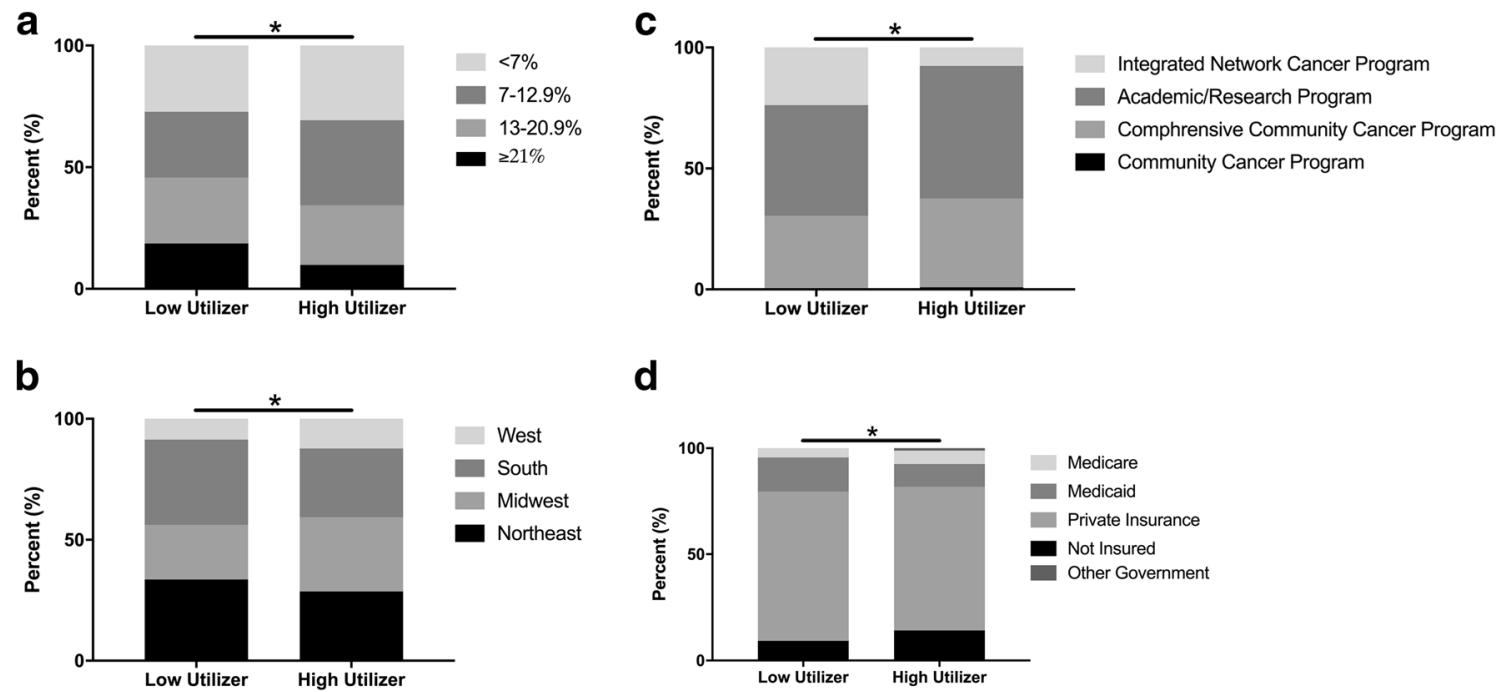

d

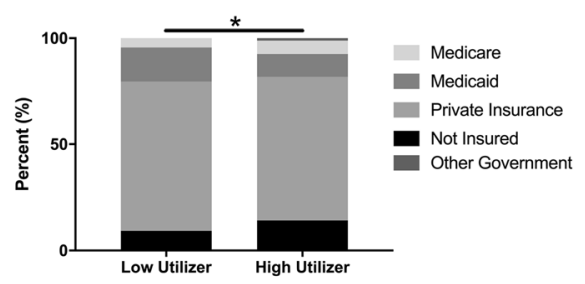

Fig. 5 a Patients treated at high-utilization hospitals were more likely to be better educated as depicted by the lower proportion of patients without a high school degree. b Higher-utilization hospitals were more likely to be located in the Midwest and West compared with low-utilization hospitals.

c High-utilization hospitals consisted of a greater proportion of academic centers and lower proportion of integrated network cancer programs. d Compared with patients treated at low-utilization hospitals, those treated at high-utilizer hospitals were more likely to be uninsured 
hospitals based on adjusted adjuvant chemotherapy use. Previous studies have shown that community and comprehensive treatment centers were more likely to give both neoadjuvant and adjuvant chemotherapy to stage II or III rectal cancer. ${ }^{10,24}$ Our study, on the other hand, did not show any difference in adjuvant chemotherapy use based on facility type. However, when adjusted, HUH were more likely to be academic centers and comprehensive community cancer programs. High-volume hospitals have also been shown to be associated with increased compliance with consensus guidelines. ${ }^{10,24}$ While we found that adjuvant chemotherapy was more commonly used in high-volume centers, we did not find any difference in surgical volume between HUH and LUH. Different definitions of high- and low-volume hospitals and changes in hospital classifications may account for these discordant findings. This data may suggest that higher volume, academic hospitals are more likely to follow consensus guidelines, but the question remains as to why there is such wide variability nationally. The use of multidisciplinary tumor boards may be more prevalent in high volume, academic centers. However, the NCDB does not contain this information and we are unfortunately unable to investigate that hypothesis. Patients forgo adjuvant chemotherapy for many reasons, including post-operative complications, comorbidities, patient preference, or physician recommendation. The NCDB is missing detailed information in regard to these variables and limits our ability to ascertain why these patients did not receive adjuvant chemotherapy. However, we did not find any difference in Charlson Comorbidity score between the groups. Hospital length of stay and unplanned readmission are the only surrogates available for complications. There was no difference in readmission rate and the slightly longer length of stay that is unlikely to be clinically significant. There is an entire field of knowledge transfer that seeks to identify barriers to the dissemination of information in healthcare, and it is estimated that it takes 17 years for information to translate to the bedside. ${ }^{25,26}$ Future studies should focus on identifying interventions to help disseminate evidence, identifying barriers to receiving adjuvant chemotherapy and improve outcomes for rectal cancer patients.

This study has several limitations. First, there are inherent biases to retrospective studies, but we have attempted to account for these through the use of multivariate and riskadjusted models. Second, the NCDB is a national database and may be prone to missing or inaccurate data similar to any other large registry. The outcomes provided in the database only allow us to calculate OS and do not contain any information regarding the cause of death. The NCDB also has limited information regarding clinical decision-making and does not allow for conclusions about why there is poor compliance with adjuvant chemotherapy. The NCDB also does not contain variables such as type of chemotherapy and pathologic factors, including mesorectal fascia involvement and extramural vascular invasion, that could be associated with outcomes. Despite these shortcomings, the NCDB consists of the majority of new U.S. cancer diagnoses and contains many cancer-related variables that are not found in other large databases. There is also a growing interest in using the TNT approach, where all chemotherapy and radiation are given before surgery. This approach may improve compliance, as chemotherapy is delivered upfront, but long-term results are not yet available. ${ }^{27-29}$ The NCDB does not delineate TNT in the database, and using the definition as described by Zhu et al., our study contains only $49(2.0 \%)$ patients that may have undergone TNT. ${ }^{13}$ Due to the low percentage and need for a surrogate definition, these patients were not removed from the cohort.

In conclusion, adjuvant chemotherapy is associated with a survival benefit in rectal cancer patients with large initial tumors and a pCR following neoadjuvant chemoradiation and oncologic resection. However, the utilization of this strategy at U.S. hospitals is currently quite low. There is significant variability in U.S. practice patterns, with the majority of higher utilizing hospitals being academic centers. Largescale efforts are needed to standardize treatment patterns according to national guidelines.

Authorship Contribution Study concept and design: Morris, Winer, Paquette; data acquisition: Morris, Paquette; data analysis and interpretation: Morris, Winer, Lee, Shah, Rafferty, Paquette; drafting and critical revision of the manuscript: Morris, Winer, Lee, Shah, Rafferty, Paquette; final approval of the manuscript: Morris, Winer, Lee, Shah, Rafferty, Paquette.

\section{Compliance with Ethical Standards}

Conflict of Interest The authors declare that they have no conflict of interest.

\section{References}

1. National Comprehensive Cancer Network. Rectal Cancer (Version 1.2020). https://www.ncen.org/professionals/physician_gls/pdf/ rectal.pdf Accessed December 29, 2019.

2. Monson, JR, Weiser, MR, Buie, WD, Chang, GJ, Rafferty, JF, Buie, WD, Rafferty, J, Standards Practice Task Force of the American Society of, C, and Rectal, S. Practice parameters for the management of rectal cancer (revised). Dis Colon Rectum 2013;56(5):535-550.

3. Garcia-Aguilar, J, Chow, OS, Smith, DD, Marcet, JE, Cataldo, PA, Varma, MG, Kumar, AS, Oommen, S, Coutsoftides, T, Hunt, SR, Stamos, MJ, Ternent, CA, Herzig, DO, Fichera, A, Polite, BN, Dietz, DW, Patil, S, Avila, K, and Timing of Rectal Cancer Response to Chemoradiation, C. Effect of adding mFOLFOX6 after neoadjuvant chemoradiation in locally advanced rectal cancer: a multicentre, phase 2 trial. Lancet Oncol 2015;16(8):957-966. 
4. Huang, Y, Lee, D, and Young, C. Predictors for complete pathological response for stage II and III rectal cancer following neoadjuvant therapy - A systematic review and meta-analysis. Am J Surg 2020

5. Glynne-Jones, R, Counsell, N, Quirke, P, Mortensen, N, Maraveyas, A, Meadows, HM, Ledermann, J, and SebagMontefiore, D. Chronicle: results of a randomised phase III trial in locally advanced rectal cancer after neoadjuvant chemoradiation randomising postoperative adjuvant capecitabine plus oxaliplatin (XELOX) versus control. Ann Oncol 2014;25(7):1356-1362.

6. Breugom, AJ, van Gijn, W, Muller, EW, Berglund, A, van den Broek, CB, Fokstuen, T, Gelderblom, H, Kapiteijn, E, Leer, JW, Marijnen, CA, Martijn, H, Meershoek-Klein Kranenbarg, E, Nagtegaal, ID, Pahlman, L, Punt, CJ, Putter, H, Roodvoets, AG, Rutten, HJ, Steup, WH, Glimelius, B, van de Velde, CJ, Cooperative Investigators of Dutch Colorectal Cancer, G, and Nordic Gastrointestinal Tumour Adjuvant Therapy, G. Adjuvant chemotherapy for rectal cancer patients treated with preoperative (chemo)radiotherapy and total mesorectal excision: a Dutch Colorectal Cancer Group (DCCG) randomized phase III trial. Ann Oncol 2015;26(4):696-701.

7. Sao Juliao, GP, Karagkounis, G, Fernandez, LM, Habr-Gama, A, Vailati, BB, Dattani, M, Kalady, MF, and Perez, RO. Conditional Survival in Patients With Rectal Cancer and Complete Clinical Response Managed by Watch and Wait After Chemoradiation: Recurrence Risk Over Time. Ann Surg 2019;

8. van der Valk, MJM, Hilling, DE, Bastiaannet, E, Meershoek-Klein Kranenbarg, E, Beets, GL, Figueiredo, NL, Habr-Gama, A, Perez, RO, Renehan, AG, van de Velde, CJH, and Consortium, I. Longterm outcomes of clinical complete responders after neoadjuvant treatment for rectal cancer in the International Watch \& Wait Database (IWWD): an international multicentre registry study. Lancet 2018;391(10139):2537-2545.

9. Turner, MC, Keenan, JE, Rushing, CN, Gulack, BC, Nussbaum, DP, Benrashid, E, Hyslop, T, Strickler, JH, Mantyh, CR, and Migaly, J. Adjuvant Chemotherapy Improves Survival Following Resection of Locally Advanced Rectal Cancer with Pathologic Complete Response. J Gastrointest Surg 2019;23(8):1614-1622.

10. $\mathrm{Xu}, \mathrm{Z}$, Mohile, SG, Tejani, MA, Becerra, AZ, Probst, CP, Aquina, CT, Hensley, BJ, Arsalanizadeh, R, Noyes, K, Monson, JR, and Fleming, FJ. Poor compliance with adjuvant chemotherapy use associated with poorer survival in patients with rectal cancer: An NCDB analysis. Cancer 2017;123(1):52-61.

11. Charlson, ME, Pompei, P, Ales, KL, and MacKenzie, CR. A new method of classifying prognostic comorbidity in longitudinal studies: development and validation. J Chronic Dis 1987;40(5):373383.

12. Deyo, RA, Cherkin, DC, and Ciol, MA. Adapting a clinical comorbidity index for use with ICD-9-CM administrative databases. J Clin Epidemiol 1992;45(6):613-619.

13. Zhu, S, Brodin, NP, English, K, Ohri, N, Chuy, JW, Rajdev, LN, Narang, R, Kalnicki, S, Guha, C, Garg, MK, and Kabarriti, R. Comparing outcomes following total neoadjuvant therapy and following neoadjuvant chemoradiation therapy in patients with locally advanced rectal cancer. EClinical Medicine 2019;16, $23-29$.

14. Daly, MC, Hanseman, DJ, Abbott, DE, Shah, SA, and Paquette, IM. Hospital Variability in Use of Adjuvant Chemotherapy for Patients with Stage 2 and 3 Colon Cancer. Dis Colon Rectum 2016;59(12):1134-1141.

15. Midura, EF, Jung, AD, Daly, MC, Hanseman, DJ, Davis, BR, Shah, SA, and Paquette, IM. Cancer Center Volume and Type Impact Stage-Specific Utilization of Neoadjuvant Therapy in Rectal Cancer. Dig Dis Sci 2017;62(8):1906-1912.

16. Dimick, JB, Ghaferi, AA, Osborne, NH, Ko, CY, and Hall, BL. Reliability adjustment for reporting hospital outcomes with surgery. Ann Surg 2012;255(4):703-707.
17. Dimick, JB, Staiger, DO, and Birkmeyer, JD. Ranking hospitals on surgical mortality: the importance of reliability adjustment. Health Serv Res 2010;45(6 Pt 1):1614-1629.

18. Dossa, F, Acuna, SA, Rickles, AS, Berho, M, Wexner, SD, Quereshy, FA, Baxter, NN, and Chadi, SA. Association Between Adjuvant Chemotherapy and Overall Survival in Patients With Rectal Cancer and Pathological Complete Response After Neoadjuvant Chemotherapy and Resection. JAMA Oncol 2018;4(7):930-937.

19. Polanco, PM, Mokdad, AA, Zhu, H, Choti, MA, and Huerta, S. Association of Adjuvant Chemotherapy With Overall Survival in Patients With Rectal Cancer and Pathologic Complete Response Following Neoadjuvant Chemotherapy and Resection. JAMA Oncol 2018;4(7):938-943.

20. Voss, RK, Lin, JC, Roper, MT, Al-Temimi, MH, Ruan, JH, Tseng, WH, Tam, M, Sherman, MJ, Klaristenfeld, DD, and Tomassi, MJ. Adjuvant Chemotherapy Does Not Improve Recurrence-Free Survival in Patients With Stage 2 or Stage 3 Rectal Cancer After Neoadjuvant Chemoradiotherapy and Total Mesorectal Excision. Dis Colon Rectum 2020;63(4):427-440.

21. Petersen, SH, Harling, H, Kirkeby, LT, Wille-Jorgensen, P, and Mocellin, S. Postoperative adjuvant chemotherapy in rectal cancer operated for cure. Cochrane Database Syst Rev 2012;3:CD004078.

22. Li, Q, Luo, D, Zhu, J, Yang, L, Liu, Q, Ma, Y, Liang, L, Cai, S, Zhang, Z, Li, X, and group, ACs. ACRNaCT trial protocol: efficacy of adjuvant chemotherapy in patients with clinical T3b/T4, N+ rectal Cancer undergoing Neoadjuvant Chemoradiotherapy: a pathology-oriented, prospective, multicenter, randomized, open-label, parallel group clinical trial. BMC Cancer 2019;19(1):1117.

23. Dong, Z, Xu, J, Wang, Z, and Petrov, MS. Stents for the prevention of pancreatic fistula following pancreaticoduodenectomy. Cochrane Database Syst Rev 2016;5:CD008914.

24. Monson, JR, Probst, CP, Wexner, SD, Remzi, FH, Fleshman, JW, Garcia-Aguilar, J, Chang, GJ, Dietz, DW, and Consortium for Optimizing the Treatment of Rectal, C. Failure of evidence-based cancer care in the United States: the association between rectal cancer treatment, cancer center volume, and geography. Ann Surg 2014;260(4):625-631; discussion 631-622.

25. Kahn, JM. Disseminating clinical trial results in critical care. Crit Care Med 2009;37(1 Suppl):S147-153.

26. Morris, ZS, Wooding, S, and Grant, J. The answer is 17 years, what is the question: understanding time lags in translational research. $\mathrm{J}$ R Soc Med 2011;104(12):510-520.

27. Fokas, E, Allgauer, M, Polat, B, Klautke, G, Grabenbauer, GG, Fietkau, R, Kuhnt, T, Staib, L, Brunner, T, Grosu, AL, Schmiegel, W, Jacobasch, L, Weitz, J, Folprecht, G, SchlenskaLange, A, Flentje, M, Germer, CT, Grutzmann, R, Schwarzbach, M, Paolucci, V, Bechstein, WO, Friede, T, Ghadimi, M, Hofheinz, RD, Rodel, C, and German Rectal Cancer Study, G. Randomized Phase II Trial of Chemoradiotherapy Plus Induction or Consolidation Chemotherapy as Total Neoadjuvant Therapy for Locally Advanced Rectal Cancer: CAO/ARO/AIO-12. J Clin Oncol 2019;37(34):3212-3222.

28. Ludmir, EB, Palta, M, Willett, CG, and Czito, BG. Total neoadjuvant therapy for rectal cancer: An emerging option. Cancer 2017;123(9):1497-1506.

29. Franke, AJ, Parekh, H, Starr, JS, Tan, SA, Iqbal, A, and George, TJ, Jr. Total Neoadjuvant Therapy: A Shifting Paradigm in Locally Advanced Rectal Cancer Management. Clin Colorectal Cancer 2018;17(1):1-12.

Publisher's Note Springer Nature remains neutral with regard to jurisdictional claims in published maps and institutional affiliations. 